\title{
Parametric testing statistical hypotheses for fuzzy random variables
}

\author{
Gholamreza Hesamian · Mehdi Shams
}

Published online: 5 February 2015

(C) The Author(s) 2015. This article is published with open access at Springerlink.com

\begin{abstract}
In this paper, a method is proposed for testing statistical hypotheses about the fuzzy parameter of the underlying parametric population. In this approach, using definition of fuzzy random variables, the concept of the power of test and $p$ value is extended to the fuzzy power and fuzzy $p$ value. To do this, the concepts of fuzzy $p$ value have been defined using the $\alpha$-optimistic values of the fuzzy observations and fuzzy parameters. This paper also develop the concepts of fuzzy type-I, fuzzy type-II errors and fuzzy power for the proposed hypothesis tests. To make decision as a fuzzy test, a well-known index is employed to compare the observed fuzzy $p$ value and a given significance value. The result provides a fuzzy test function which leads to some degrees to accept or to reject the null hypothesis. As an application of the proposed method, we focus on the normal fuzzy random variable to investigate hypotheses about the related fuzzy parameters. An applied example is provided throughout the paper clarifying the discussions made in this paper.
\end{abstract}

Keywords $\alpha$-Optimistic value $\cdot$ Fuzzy random variable . Fuzzy normal variable · Fuzzy parameter - Fuzzy power test · Fuzzy type-I error · Fuzzy type-II error

Communicated by V. Loia.

G. Hesamian $(\varangle)$

Department of Statistics, Payame Noor University,

19395-3697 Tehran, Iran

e-mail: gh.hesamian@pnu.ac.ir

\section{Shams}

Department of Mathematical Sciences,

University of Kashan, Isfahan, Iran

e-mail: mehdishams@kashanu.ac.ir

\section{Introduction}

The purpose of statistical inference is to draw conclusions about a population on the basis of data obtained from a sample of that population. Hypothesis testing is the process used to evaluate the strength of evidence from the sample and provides a framework for making decisions related to the population, i.e., it provides a method for understanding how reliably one can extrapolate observed findings in a sample under study to the larger population from which the sample was drawn. The investigator formulates a specific hypothesis, evaluates data from the sample, and uses these data to decide whether they support the specific hypothesis. The classical parametric approaches usually depend on certain basic assumptions about the underlying population such as: crisp observations, exact parameters, crisp hypotheses, and crisp possible decisions. In practical studies, however, it is frequently difficult to assume that the parameter, for which the distribution of a random variable is determined, has a precise value or the value of the random variable is recorded as a precise value or the hypotheses of interest are presented as exact relations, and so on. Therefore, to achieve suitable testing statistical methods dealing with imprecise information, we need to model the imprecise information and extend the usual approaches to imprecise environments. Since its introduction by Zadeh (1965), fuzzy set theory has been developed and applied in some statistical contexts to deal with uncertainty conditions such as above situations. Specially, the topic of testing statistical hypotheses in fuzzy environments has extensively been studied. Below is a brief review of some studies relevant to the present work. Arnold (1996, 1998) presented an approach for testing fuzzily formulated hypotheses based on crisp data, in which he proposed and considered generalized definitions of the probabilities of the errors of type-I and type-II. Viertl $(2006,2011)$ used the 
extension principle to obtain the generalized estimators for a crisp parameter based on fuzzy data. He also developed some other statistical inferences for the crisp parameter, such as generalized confidence intervals and $p$ value, based on fuzzy data. Taheri and Behboodian (1999) formulated the problem of testing fuzzy hypotheses when the observations are crisp. They presented some definitions for the probabilities of type-I and type-II errors, and proved an extended version of the Neyman-Pearson Lemma. Their approach has been extended by Torabi et al. (2006) to the case in which the data are fuzzy, too. Taheri and Behboodian (2001) also studied the problem of testing hypotheses from a Bayesian point of view when the observations are ordinary and the hypotheses are fuzzy. Taheri and Arefi (2009) presented an approach to the problem of testing fuzzy hypotheses, based on the so-called fuzzy critical regions. Grzegorzewski (2000) suggested some fuzzy tests for crisp hypotheses concerning an unknown parameter of a population using fuzzy random variables (FRVs). Montenegro et al. (2001, 2004), using a generalized metric for fuzzy numbers, proposed a method to test hypotheses about the fuzzy mean of a FRV in one and two populations settings. Gonzalez-Rodríguez et al. (2006) extended a one-sample bootstrap method of testing about the mean of a general fuzzy random variable. Gil et al. (2006) introduced a bootstrap approach to the multiple-sample test of means for imprecisely valued sample data. Chachi and Taheri (2011) introduced a new approach to construct fuzzy confidence intervals for the fuzzy mean of a FRV. Filzmoser and Viertl (2004) and Parchami et al. (2010) presented $p$ value-based approaches to the problem of testing hypothesis, when the available data or the hypotheses of interest are fuzzy, respectively. Hryniewicz (2006b) investigated the concept of $p$ value in a possibilistic context in which the concept of $p$ value is generalized for the case of imprecisely defined statistical hypotheses and vague statistical data.

On the other hand, there have been some studies on non-parametric statistical testing hypotheses in fuzzy environment. Concerning the purposes of this paper, let us briefly review some of the literature on this topic. Kahraman et al. (2004) proposed some algorithms for fuzzy non-parametric rank-sum tests based on fuzzy random variables. Grzegorzewski (1998) introduced a method to estimate the median of a population using fuzzy random variables. He (Grzegorzewski 2004) also demonstrated a straightforward generalization of some classical non-parametric tests for fuzzy random variables based on a metric in the space of fuzzy numbers. He also (Grzegorzewski 2005, 2009) studied some non-parametric median fuzzy tests for fuzzy observations showing a degree of possibility and a degree of necessity (Dubois and Prade 1983) for evaluating the underlying hypotheses. In addition, he (Grzegorzewski 2008) proposed a modification of the classical sign test to cope with fuzzy data which was so-called bi-robust test, i.e., a test which is both distribution free and which does not depend so heavily on the shape of the membership functions used for modeling fuzzy data. Denœux et al. (2005), using a fuzzy partial ordering on closed intervals, extended the non-parametric ranksum tests based on fuzzy data. For evaluating the hypotheses of interest at a crisp or a fuzzy significance level, they employed the concepts of fuzzy $p$ value and degree of rejection of the null hypothesis quantified by a degree of possibility and a degree of necessity. Hryniewicz (2006a) investigated the fuzzy version of the Goodman-Kruskal $\gamma$-statistic described by ordered categorical data. Lin et al. (2010) considered the problem of two-sample Kolmogorov-Smirnov test for continuous fuzzy intervals based on a crisp test statistic. Taheri and Hesamian (2011) introduced a fuzzy version of the Goodman-Kruskal $\gamma$-statistic for two-way contingency tables when the observations were crisp, but the categories were described by fuzzy sets. In this approach, a method was also developed for testing of independence in the two-way contingency tables. Taheri and Hesamian (2012) extended the Wilcoxon signed-rank test to the case where the available observations are imprecise and underlying hypotheses are crisp. Hesamian and Chachi (2013) developed the concepts of fuzzy cumulative distribution function and fuzzy empirical cumulative distribution function and investigated the large sample property of the classical empirical cumulative distribution function for fuzzy empirical cumulative distribution function. They proposed a method for developing two-sample Kolmogorov-Smirnov test for the case when the data are observations of fuzzy random variables, and the hypotheses are imprecise. For more on fuzzy statistics including testing hypotheses for imprecise data, see for example Bertoluzza et al. (2002), Buckley (2006), Kruse and Meyer (1987), Nguyen and Wu (2006), Viertl (2011).

This paper develops an approach to test hypotheses for an unknown fuzzy parameter based on fuzzy random variables. To do this, we extend the concept of fuzzy power function and fuzzy $p$ value to investigate the hypotheses of interest. Finally, a decision rule is suggested to accept or reject the null and alternative hypotheses. We also provide a computational procedure and an example to express the proposed method to test statistical hypotheses for a normal FRV.

This paper is organized as follows: Section 2 briefly reviews the classical parametric testing hypotheses and some definitions from fuzzy numbers. In the same section, some results about $\alpha$-optimistic values of a fuzzy number are derived to recontract a so-called definition of fuzzy random variables introduced in Sect. 3. In Sect. 4, one-sided and two-sided hypotheses about a fuzzy parameter of a FRV are defined. The concept of fuzzy power and fuzzy $p$ value for testing hypotheses about a fuzzy parameter of a continuous parametric population is also introduced. Then, the proposed method is applied for testing hypotheses about the fuzzy parameters of a normal FRV in Sect. 4.1. A numerical example 
is then provided in Sect. 5 to clarify the discussions made in this paper. Section 6 compares the proposed method to the other similar existing methods. Finally, a brief conclusion is provided in Sect. 7. In addition, the proofs of the main results in this paper are provided in Appendix.

\section{Preliminaries}

2.1 Testing statistical hypotheses: the classical approach

Let $\mathbf{X}=\left(X_{1}, \ldots, X_{n}\right)$ be a random sample, with the observed value $\mathbf{x}=\left(x_{1}, \ldots, x_{n}\right)$, from a continuous population with density function $f_{\boldsymbol{\theta}}$ where $\boldsymbol{\theta} \in \Theta \subseteq \mathbb{R}^{p}, p \geq 1$. The decision rule to test the null hypothesis $H_{0}: \theta \in \Theta_{0}$ versus the alternative $H_{1}: \theta \in \Theta_{1}$ is typically denoted by

$\varphi(\mathbf{x})= \begin{cases}1 & \text { if } T(\mathbf{x}) \in \mathbf{C}_{\delta}, \\ 0 & \text { if } T(\mathbf{x}) \notin \mathbf{C}_{\delta},\end{cases}$

where $\mathbf{C}_{\delta} \subseteq \mathbb{R}$ is the critical region, $\delta$ is the significance level, and $T(\mathbf{X})$ is a test statistic. The power function of $\varphi(\mathbf{X})$ is defined by $\pi_{\varphi}(\theta)=E_{\theta}[\varphi(\mathbf{X})]=\mathbf{P}_{\theta}\left(T(\mathbf{X}) \in \mathbf{C}_{\delta}\right)$. Note that, if the underlying family of distribution functions has the MLR property (Monotone Likelihood Ratio property) in $T$ then $\pi_{\varphi}(\theta)=E_{\theta}[\varphi(\mathbf{X})]$ is a non-decreasing function of $\theta$ (for more see Lehmann and Romano 2005; Shao 2003). Finally, at a given significance level $\delta$, the hypothesis $H_{0}$ is completely rejected if and only if $p$ value $<\delta$, where $p$ value $=\inf \left\{\delta \in[0,1]: T(\mathbf{x}) \in \mathbf{C}_{\delta}\right\}$ (Shao 2003). So, to accept or reject the null hypothesis, we have a test as follows

$\psi(\mathbf{x})= \begin{cases}1 & \text { if } p \text { value }<\delta, \\ 0 & \text { if } p \text { value } \geq \delta .\end{cases}$

\subsection{Fuzzy numbers}

Let $\mathbb{R}$ be the set of all real numbers. A fuzzy set of $\mathbb{R}$ is a mapping $\mu_{\widetilde{A}}: \mathbb{R} \rightarrow[0,1]$, which assigns to each $x \in \mathbb{R}$ a degree of membership $0 \leq \mu_{\widetilde{A}}(x) \leq 1$. For each $\alpha \in(0,1]$, the subset $\left\{x \in \mathbb{R} \mid \mu_{\widetilde{A}}(x) \geq \alpha\right\}$ is called the level set or $\alpha$-cut of $\widetilde{A}$ and is denoted by $\widetilde{A}[\alpha]$. The set $\widetilde{A}[0]$ is also defined equal to the closure of $\left\{x \in \mathbb{R} \mid \mu_{\widetilde{A}}(x)>0\right\}$. A fuzzy set $\widetilde{A}$ of $\mathbb{R}$ is called a fuzzy number if it satisfies the following three conditions.

1. For each $\alpha \in[0,1]$, the set $\widetilde{A}[\alpha]$ is a compact interval, which will be denoted by $\left[\widetilde{A}_{\alpha}^{L}, \widetilde{A}_{\alpha}^{U}\right]$. Here, $\widetilde{A}_{\alpha}^{L}=\inf \{x \in$ $\left.\mathbb{R} \mid \mu_{\widetilde{A}}(x) \geq \alpha\right\}$ and $\widetilde{A}_{\alpha}^{U}=\sup \left\{x \in \mathbb{R} \mid \mu_{\widetilde{A}}(x) \geq \alpha\right\}$.

2. For $\alpha, \beta \in[0,1]$, with $\alpha<\beta, \widetilde{A}[\beta] \subseteq \widetilde{A}[\alpha]$.

3. There is a unique real number $x^{*}=x_{\widetilde{A}}^{*} \in \mathbb{R}$, such that $\widetilde{A}\left(x^{*}\right)=1$. Equivalently, the set $\widetilde{A}[1]$ is a singleton.

The set of all fuzzy numbers is denoted by $\mathcal{F}(\mathbb{R})$. Moreover, we denote by $\mathcal{F}_{c}(\mathbb{R})$ the set of all fuzzy numbers with continuous membership function.
Lemma 1 (Lee 2005) Let $\widetilde{A} \in \mathcal{F}(\mathbb{R})$. Then, both the maps $\alpha \mapsto \widetilde{A}_{\alpha}^{L}$ and $\alpha \mapsto \widetilde{A}_{\alpha}^{U}$ are left continuous on $[0,1]$.

A $L R$-fuzzy number $\widetilde{A}=\left(a, a^{l}, a^{r}\right)_{L R}$ where $a^{l}, a^{r} \geq 0$, is defined as follows

$\mu_{\widetilde{A}}(x)= \begin{cases}L\left(\frac{a-x}{a^{l}}\right) & a-a^{l} \leq x \leq a, \\ R\left(\frac{x-a}{a^{r}}\right) & a<x \leq a+a^{r},\end{cases}$

where $L$ and $R$ are continuous and strictly decreasing functions with $L(0)=R(0)=1$ and $L(1)=R(1)=0$ (Lee 2005). A special type of $L R$-fuzzy numbers is the socalled triangular fuzzy numbers with the shape functions $L(x)=R(x)=\max \{0,1-|x|\}, x \in \mathbb{R}$.

A well-known ordering of fuzzy numbers, used in the sections below for defining the hypotheses of interest is defined as follows:

Definition 1 (Wu 2005) Let $\widetilde{A}, \widetilde{B} \in \mathcal{F}(\mathbb{R})$, then

1. $\widetilde{A} \asymp(\neq) \widetilde{B}$, if $\widetilde{A}_{\alpha}^{L}=(\neq) \widetilde{B}_{\alpha}^{L}$ and $\widetilde{A}_{\alpha}^{U}=(\neq) \widetilde{B}_{\alpha}^{U}$ for any $\alpha \in[0,1]$.

2. $\widetilde{A} \preceq(\prec) \widetilde{B}$, if $\widetilde{A}_{\alpha}^{L} \leq(<) \widetilde{B}_{\alpha}^{L}$ and $\widetilde{A}_{\alpha}^{U} \leq(<) \widetilde{B}_{\alpha}^{U}$ for any $\alpha \in[0,1]$.

3. $\widetilde{A} \succeq(\succ) \widetilde{B}$, if $\widetilde{A}_{\alpha}^{L} \geq(>) \widetilde{B}_{\alpha}^{L}$ and $\widetilde{A}_{\alpha}^{U} \geq(>) \widetilde{B}_{\alpha}^{U}$ for any $\alpha \in[0,1]$.

\section{$2.3 \alpha$-Optimistic values}

In this subsection, we drive some results about the $\alpha$ optimistic values of a fuzzy number. We will use these results to reconstruct a definition of fuzzy random variable that we use in the next sections.

For a given fuzzy number $\widetilde{A}$, the credibility of the event $\{\widetilde{A} \geq r\}$ is defined by Liu (2004) as follows:

$$
\operatorname{Cr}\{\widetilde{A} \geq r\}:=\frac{1}{2}\left(\sup _{y \in[r,+\infty)} \mu_{\widetilde{A}}(y)+1-\sup _{y \in(-\infty, r)} \mu_{\widetilde{A}}(y)\right),
$$

It is worth noting that: (1) $\operatorname{Cr}\{\widetilde{A} \geq r\} \in[0,1]$ and (2) $\operatorname{Cr}\{\widetilde{A} \geq$ $r\}=1-\operatorname{Cr}\{\tilde{A}<r\}$.

Here, we recall the definition of the $\alpha$-optimistic, but with a small change in the structure of the original definition. For a fuzzy number $\widetilde{A}$ and the real number $\alpha \in[0,1]$, the $\alpha$ optimistic value of $\widetilde{A}$, denoted by $\widetilde{A}_{\alpha}$, is rewritten by

$\widetilde{A}_{\alpha}:=\sup \{x \in \widetilde{A}[0] \mid \operatorname{Cr}\{\widetilde{A} \geq x\} \geq \alpha\}$.

Remark 1 It is mentioned that, according to the Liu's definition, the $\alpha$-optimistic value of the fuzzy number $\widetilde{A}$ is defined as follows

$\widetilde{A}_{\alpha}:=\sup \{x \in \mathbb{R} \mid \operatorname{Cr}\{\tilde{A} \geq x\} \geq \alpha\}$.

Therefore, we observe that $\widetilde{A}_{0}=\infty$ and $\widetilde{A}_{\alpha} \in\left[\widetilde{A}_{\alpha}^{L}, \widetilde{A}_{\alpha}^{R}\right)$ for $\alpha \in(0,1]$. While, by Eq. (2), each value of $\widetilde{A}_{\alpha}$ belongs to 
$\widetilde{A}[0]$ (which is a compact interval, due to the definition of a fuzzy number), for all $\alpha \in[0,1]$. Moreover, it is clear that $\widetilde{A}_{\alpha}$ is a non-increasing function of $\alpha \in[0,1]$ (see Liu 2004, for more details).

Example 1 For a given $L R$-fuzzy number $\widetilde{A}=\left(a, a^{l}, a^{r}\right)$, it is easily seen that

$\widetilde{A}_{\alpha}= \begin{cases}a+a^{r} R^{-1}(2 \alpha) & \text { for } 0.0 \leq \alpha \leq 0.5 \\ a-a^{l} L^{-1}(2(1-\alpha)) & \text { for } 0.5 \leq \alpha \leq 1.0\end{cases}$

Specially, if $\widetilde{A}=\left(a, a^{l}, a^{r}\right)_{T}$ is a triangular fuzzy number, then

$\widetilde{A}_{\alpha}= \begin{cases}a^{r}-2 \alpha\left(a^{r}-a\right) & \text { for } 0.0 \leq \alpha \leq 0.5, \\ 2 a-a^{l}-2 \alpha\left(a-a^{l}\right) & \text { for } 0.5 \leq \alpha \leq 1.0 .\end{cases}$

For instance, the $\alpha$-optimistic values of $\widetilde{A}=(-2,0,1)_{T}$ are obtained as

$\widetilde{A}_{\alpha}= \begin{cases}1-2 \alpha & \text { for } 0.0 \leq \alpha \leq 0.5 \\ 2-4 \alpha & \text { for } 0.5 \leq \alpha \leq 1.0 .\end{cases}$

In the rest of this section, we drive some properties of $\alpha$ optimistic values of a fuzzy number $\widetilde{A}$ and its relation to $\alpha$-cuts of $\tilde{A}$.

Theorem 1 For $\widetilde{A} \in \mathcal{F}(\mathbb{R})$, suppose $\phi_{\widetilde{A}}:[0,1] \rightarrow \mathbb{R}$ is the function defined by $\phi_{\widetilde{A}}(\alpha)=\widetilde{A}_{\alpha}$. Then,

(i) For each $\alpha \in[0,1]$,

$$
\begin{aligned}
& \phi_{\widetilde{A}}(\alpha) \\
& \quad= \begin{cases}\widetilde{A}_{2 \alpha}^{U} & 0.0 \leq \alpha \leq 0.5, \\
\sup \left\{x \leq x^{*} \mid \mu_{\widetilde{A}}(x) \leq 2(1-\alpha)\right\} & 0.5<\alpha \leq 1,\end{cases}
\end{aligned}
$$

where $x^{*}=x_{\widetilde{A}}^{*} \in \mathbb{R}$ is the unique real number which satisfies $\widetilde{A}\left(x^{*}\right)=1$.

(ii) $\phi_{\widetilde{A}}$ is a decreasing and left continuous function.

Proof The proof of Theorem 1 is postponed to the Appendix.

Here, we investigate another useful property of the function $\phi_{\widetilde{A}}$. For a fuzzy number $\widetilde{A} \in \mathcal{F}(\mathbb{R})$, let $\psi_{\widetilde{A}}:[0,1] \rightarrow \mathbb{R}$ be a function defined as follows.

$\forall \alpha \in[0,1], \quad \psi_{\widetilde{A}}(\alpha)= \begin{cases}\widetilde{A}_{2 \alpha}^{U} & \alpha \in[0,0.5], \\ \widetilde{A}_{2(1-\alpha)}^{L} & \alpha \in(0.5,1] .\end{cases}$

According to Lemma 1, this function is decreasing, left continuous on $[0,0.5]$ and right continuous on $(0.5,1]$. Moreover, according to part (i) of the previous theorem, $\psi_{\widetilde{A}}(\alpha)=$ $\phi_{\widetilde{A}}(\alpha)$, for all $\alpha \in[0,0.5]$.

Lemma 2 If $\alpha_{0} \in(0.5,1]$ is a point of continuity of either $\psi_{\widetilde{A}}$ or $\phi_{\widetilde{A}}$ then the values of the two functions coincide at this point.

Proof The proof of Lemma 2 is postponed to the Appendix.
Remark 2 From Lemma 2, note that the $\alpha$-cuts of a fuzzy number $\widetilde{A} \in \mathcal{F}_{c}(\mathbb{R})$ can be rewritten as $\widetilde{A}[\alpha]=\left[\widetilde{A}_{1-\alpha / 2}\right.$, $\left.\widetilde{A}_{\alpha / 2}\right], \alpha \in[0,1]$. Therefore, the membership function of the fuzzy number $\widetilde{A}$ can be obtained from the representation theorem (e.g., see Lee 2005) as follows

$\mu_{\widetilde{A}}(x)=\sup _{\alpha \in[0,1]} I\left(x \in\left[\widetilde{A}_{1-\alpha / 2}, \widetilde{A}_{\alpha / 2}\right]\right), \quad x \in \mathbb{R}$.

Remark 3 From Lemma 2, note that the ordering of fuzzy numbers $\widetilde{A}$ and $\widetilde{B}$ introduced in Definition 1 is equivalent to:

1. $\widetilde{A} \asymp(\neq) \widetilde{B}$, if $\widetilde{A}_{\alpha}=(\neq) \widetilde{B}_{\alpha}$ for any $\alpha \in[0,1]$.

2. $\widetilde{A} \preceq(\prec) \widetilde{B}$, if $\widetilde{A}_{\alpha} \leq(<) \widetilde{B}_{\alpha}$ for any $\alpha \in[0,1]$.

3. $\widetilde{A} \succeq(\succ) \widetilde{B}$, if $\widetilde{A}_{\alpha} \geq(>) \widetilde{B}_{\alpha}$ for any $\alpha \in[0,1]$.

\section{Fuzzy random variables}

Let $(\Omega, \mathcal{A}, \mathbf{P})$ be a probability space, $\tilde{X}: \Omega \rightarrow \mathcal{F}(\mathbb{R})$ be a fuzzy-valued function, $X$ be a random variable having distribution function $f_{\boldsymbol{\theta}}$ with a vector of parameters $\boldsymbol{\theta}=\left(\theta_{1}, \ldots, \theta_{p}\right) \in \Theta$ in which $\Theta \subset \mathbb{R}^{p}$ be the parameter space, $p \geq 1$. Throughout this paper, we assume that all random variables have the same probability space $(\Omega, \mathcal{A}, \mathbf{P})$.

It is mentioned that Kwakernaak (1978, 1979) introduced a notion of FRVs as follows: given a probability space $(\Omega, \mathcal{A}, \mathbf{P})$, a mapping $\tilde{X}: \Omega \rightarrow \mathcal{F}(\mathbb{R})$ is said to be a FRV if for all $\alpha \in[0,1]$, the two real-valued mappings $\widetilde{X}_{\alpha}^{L}: \Omega \rightarrow \mathbb{R}$ and $\widetilde{X}_{\alpha}^{U}: \Omega \rightarrow \mathbb{R}$ are real-valued random variables. Here, we rewrite the Kwakernaak's definition of FRV using $\alpha$-optimistic values of a fuzzy number as follows

Definition 2 (Hesamian and Chachi 2013) The fuzzy-valued function $\tilde{X}: \Omega \rightarrow \mathcal{F}(\mathbb{R})$ is called a FRV if $\tilde{X}_{\alpha}(\omega): \Omega \rightarrow \mathbb{R}$ is a real-valued random variable for all $\alpha \in[0,1]$.

Based on Theorem 1 and Remark 2, note that our definition of a FRV is just a restriction of FRVs defined by Kwakernaak. So, a FRV can be addressed by its $\alpha$-optimistic values instead of its $\alpha$-cuts which is the reason that we use credibility index in this paper.

Definition 3 FRVs $\widetilde{X}$ and $\widetilde{Y}$ are called identically distributed if $\widetilde{X}_{\alpha}$ and $\widetilde{Y}_{\alpha}$ are identically distributed, for all $\alpha \in[0,1]$. In addition, they are called independent if each random variable $\widetilde{X}_{\alpha}$ is independent of each random variable $\widetilde{Y}_{\alpha}$, for all $\alpha \in[0,1]$. Similarly, we say that $\widetilde{\mathbf{X}}=\left(\widetilde{X}_{1}, \ldots, \widetilde{X}_{n}\right)$ is a random sample of size $n$ if $\widetilde{X}_{i}$ are independent and identically distributed FRVs. The observed fuzzy random sample is denoted by $\widetilde{\mathbf{x}}=\left(\widetilde{x}_{1}, \ldots, \widetilde{x}_{n}\right)$.

Here, consider a special case of FRVs as the member of a family of a classical parametric population which is introduced by Wu (2005) (see also Chachi and Taheri 2011). 
Definition 4 A FRV $\widetilde{X}$ is said to have a (classical) parametric continuous density function with fuzzy parameter $\widetilde{\boldsymbol{\theta}}: \Theta \rightarrow(\mathcal{F}(\mathbb{R}))^{p}$, denoted by $\left\{f_{\widetilde{\boldsymbol{\theta}}}: \widetilde{\boldsymbol{\theta}} \in(\mathcal{F}(\mathbb{R}))^{p}\right\}$, if for all $\alpha \in[0,1], \widetilde{X}_{\alpha} \sim f_{(\widetilde{\boldsymbol{\theta}})_{\alpha}}$, where $(\widetilde{\boldsymbol{\theta}})_{\alpha}=\left(\left(\widetilde{\theta}_{1}\right)_{\alpha}, \ldots,\left(\widetilde{\theta}_{p}\right)_{\alpha}\right)$.

Remark 4 It is mentioned that a necessary and sufficient condition to say $\widetilde{X} \sim f_{\widetilde{\boldsymbol{\theta}}}, \widetilde{\boldsymbol{\theta}} \in(\mathcal{F}(\mathbb{R}))^{p}$ is that $\widetilde{X}_{\alpha_{1}}$ is "stochastically greater than $\widetilde{X}_{\alpha_{2}}$ for any $\alpha_{1}<\alpha_{2}$, i.e., $F_{\widetilde{X}_{\alpha_{2}}}(x) \geq F_{\widetilde{X}_{\alpha_{1}}}(x)$ for all $x \in \mathbb{R}$. For instance, let $\widetilde{X}_{\alpha} \sim$ $N\left(\widetilde{\mu}_{1-\alpha},{\widetilde{\sigma^{2}}}_{1-\alpha}\right)$, where $\widetilde{\mu} \in \mathcal{F}(\mathbb{R})$ and $\widetilde{\sigma^{2}} \in \mathcal{F}((0, \infty))$. Then, it is easy to verify that $F_{\widetilde{X}_{\alpha_{2}}}(x) \geq F_{\widetilde{X}_{\alpha_{1}}}(x)$ for all $x \in \mathbb{R}$ and for every $\alpha_{1}<\alpha_{2}$. So, $\tilde{X}$ is said to be normally distributed with fuzzy parameters $\tilde{\mu}$ and $\widetilde{\sigma}^{2}$ if $\widetilde{X}_{\alpha} \sim N\left(\widetilde{\mu}_{1-\alpha}, \widetilde{\sigma^{2}}{ }_{1-\alpha}\right)$. As another example, assume that $\widetilde{X}_{\alpha} \sim \exp \left(\widetilde{\lambda}_{1-\alpha}\right)$ where $\tilde{\lambda} \in \mathcal{F}(0, \infty)$. Then, one can observe that $F_{\widetilde{X}_{\alpha_{2}}}(x) \geq F_{\widetilde{X}_{\alpha_{1}}}(x)$ for all $x \in \mathbb{R}$ and for every $\alpha_{1}<\alpha_{2}$ where $F_{\lambda}(x)=1-e^{-\lambda x}, x>0$. So, $\widetilde{X} \sim \exp (\widetilde{\lambda})$ if $\widetilde{X}_{\alpha} \sim \exp \left(\widetilde{\lambda}_{1-\alpha}\right)$ for all $\alpha \in[0,1]$.

Remark 5 Based on definition of a normal fuzzy random variable introduced by $\mathrm{Wu}$ (2005), a fuzzy random variable $\widetilde{X}$ is called normally distributed with fuzzy parameters $\tilde{\mu}$ and $\widetilde{\sigma^{2}}$ if and only if $\widetilde{X}_{\alpha}^{L} \sim N\left(\widetilde{\mu}_{\alpha}^{L},\left(\sigma^{2}\right)_{\alpha}^{L}\right)$ and $\widetilde{X}_{\alpha}^{U} \sim N\left(\widetilde{\mu}_{\alpha}^{U},\left(\widetilde{\sigma^{2}}\right)_{\alpha}^{U}\right)$. However, it is easy to verify that " $\widetilde{X}_{\alpha}^{U}$ is not stochastically greater than $\widetilde{X}_{\alpha}^{L}$ " for any $\alpha \in[0,1]$. But, as it noted in Remark 4, we modified the definition of a normal fuzzy random variable using the concepts of $\alpha$-optimistic values.

Now, based on a fuzzy random sample $\widetilde{\mathbf{X}}=\left(\widetilde{X}_{1}, \ldots, \widetilde{X}_{n}\right)$, we are going to generalize the classical parametric tests for FRVs.

\section{Testing hypotheses for FRVs}

In this section, based on fuzzy random sample, we develop a procedure for testing statistical hypothesis. In fact, we wish to test the following hypotheses:

Definition 5 Let $\widetilde{X}$ be a FRV from a continuous parametric population with fuzzy parameter $\tilde{\tau}$. Then:

1. Any hypothesis of the form

$$
\widetilde{H}: \tilde{\tau} \asymp \tilde{\tau}_{0} \equiv \widetilde{H}:(\tilde{\tau})_{\alpha}=\left(\tilde{\tau}_{0}\right)_{\alpha} \text { for all } \alpha \in[0,1],
$$

is called a simple hypothesis.

2. Any hypothesis of the form

$$
\widetilde{H}: \tilde{\tau} \succ \tilde{\tau}_{0} \equiv \widetilde{H}:(\tilde{\tau})_{\alpha}>\left(\tilde{\tau}_{0}\right)_{\alpha} \text { for all } \alpha \in[0,1],
$$

is called a right one-sided hypothesis.
3. Any hypothesis of the form

$$
\widetilde{H}: \tilde{\tau} \prec \tilde{\tau}_{0} \equiv \widetilde{H}:(\tilde{\tau})_{\alpha}<\left(\tilde{\tau}_{0}\right)_{\alpha} \text { for all } \alpha \in[0,1],
$$

is called a left one-sided hypothesis.

4. Any hypothesis of the form

$$
\widetilde{H}: \tilde{\tau} \neq \tilde{\tau}_{0} \equiv \widetilde{H}:(\tilde{\tau})_{\alpha} \neq\left(\tilde{\tau}_{0}\right)_{\alpha} \text { for all } \alpha \in[0,1],
$$

is called to be a two-sided hypothesis.

In the following, by introducing some definitions and lemmas, we propose a procedure to investigate a hypothesis about the fuzzy parameters of the population. To do this, we will develop the concepts of fuzzy type-I and fuzzy type-II errors, fuzzy power and fuzzy $p$ value for $\mathbf{F R V}$.

Definition 6 Let $\varphi$ be the classical test function for testing $H_{0}: \tau=\tau_{0}$ against one-sided $H_{1}: \tau>(<) \tau_{0}$ (or two-sided $\left.H_{1}: \tau \neq \tau_{0}\right)$ alternative hypothesis. For testing $\widetilde{H}_{0}: \tilde{\tau} \asymp \tilde{\tau}_{0}$ against the alternative hypothesis introduced in Definition 5 , the test statistic, based on a random sample $\widetilde{\mathbf{X}}$, is defined to be the fuzzy set $\widetilde{\varphi}(\widetilde{\mathbf{X}})$ with $\alpha$-cuts $\left[(\widetilde{\varphi})_{\alpha}^{L}(\widetilde{\mathbf{X}}),(\widetilde{\varphi})_{\alpha}^{U}(\widetilde{\mathbf{X}})\right]$, where

$$
(\widetilde{\varphi})_{\alpha}^{L}(\widetilde{\mathbf{X}})=\inf _{\beta \geq \alpha}\left(\varphi\left(\widetilde{\mathbf{X}}_{\beta}\right)\right), \quad(\widetilde{\varphi})_{\alpha}^{L}(\widetilde{\mathbf{X}})=\sup _{\beta \geq \alpha}\left(\varphi\left(\widetilde{\mathbf{X}}_{\beta}\right)\right),
$$

in which $\widetilde{\mathbf{X}}_{\beta}=\left(\left(\widetilde{X}_{1}\right)_{\beta},\left(\widetilde{X}_{2}\right)_{\beta}, \ldots,\left(\widetilde{X}_{n}\right)_{\beta}\right)$.

Definition 7 Let $\widetilde{\varphi}(\widetilde{\mathbf{X}})$ be the fuzzy test function for testing $\widetilde{H}_{0}: \tilde{\tau} \asymp \tilde{\tau}_{0}$ against the alternative hypothesis introduced in Definition 5. The power of $\widetilde{\varphi}$ in $\widetilde{\tau}^{*}$ is defined to be the fuzzy set $\widetilde{\pi}_{\widetilde{\varphi}}\left(\widetilde{\tau}^{*}\right)$ with $\alpha$-cuts $\left[\left(\widetilde{\pi}_{\tilde{\varphi}}\left(\widetilde{\tau}^{*}\right)_{\alpha}^{L},\left(\tilde{\pi}_{\tilde{\varphi}}\left(\widetilde{\tau}^{*}\right)\right)_{\alpha}^{U}\right]\right.$, where

$$
\begin{aligned}
\left(\widetilde{\pi}_{\widetilde{\varphi}}\left(\widetilde{\tau}^{*}\right)\right)_{\alpha}^{L} & =\inf _{\beta \geq \alpha} E_{H^{\beta}: \widetilde{\tau}_{\beta}=\left(\widetilde{\tau}^{*}\right)_{\beta}}\left[\varphi\left(\widetilde{\mathbf{X}}_{\beta}\right)\right],\left(\tilde{\pi}_{\tilde{\varphi}}\left(\widetilde{\tau}^{*}\right)\right)_{\alpha}^{U} \\
& =\sup _{\beta \geq \alpha} E_{H^{\beta}: \widetilde{\tau}_{\beta}=\left(\widetilde{\tau}^{*}\right)_{\beta}}\left[\varphi\left(\widetilde{\mathbf{X}}_{\beta}\right)\right],
\end{aligned}
$$

and $\widetilde{\mathbf{X}}_{\beta}=\left(\left(\widetilde{X}_{1}\right)_{\beta},\left(\widetilde{X}_{2}\right)_{\beta}, \ldots,\left(\widetilde{X}_{n}\right)_{\beta}\right), E_{H}[T]$ denotes the expectation of $T$ given $H$.

Lemma 3 Assume that $\widetilde{\boldsymbol{X}}=\left(\widetilde{X}_{1}, \ldots, \widetilde{X}_{n}\right)$ is a fuzzy random sample from a continuous family of population $\left\{f_{\tilde{\tau}}(x) ; x \in\right.$ $\mathbb{R}, \widetilde{\tau} \in \widetilde{\Theta}\}$. If the family of density functions $\left\{f_{\tau}(x) ; x \in\right.$ $\mathbb{R}, \tau \in \Theta\}$ has the MLR property in $T$ and $\varphi$ is a nonincreasing function of $T$, then $\tilde{\pi}_{\tilde{\varphi}}$ is a non-increasing function of $\widetilde{\tau}$, i.e., for every $\widetilde{\tau}_{1}, \widetilde{\tau}_{2} \in \widetilde{\Theta}$ where $\widetilde{\tau}_{1} \prec \widetilde{\tau}_{2}$, we have $\tilde{\pi}_{\tilde{\varphi}}\left(\widetilde{\tau}_{1}\right) \preceq \widetilde{\pi}_{\tilde{\varphi}}\left(\widetilde{\tau}_{2}\right)$.

Proof The proof is postponed to the Appendix.

Definition 8 Consider testing hypothesis

$\widetilde{H}_{0}: \tilde{\tau}=\tilde{\tau}_{0}$ versus $\widetilde{H}_{1}: \tilde{\tau}=\tilde{\tau}_{1}$,

in which, it is assumed that $\tilde{\tau}_{0} \prec \tilde{\tau}_{1}$ or $\tilde{\tau}_{0} \succ \tilde{\tau}_{1}$. Then, $\tilde{\pi}_{\tilde{\varphi}}\left(\tilde{\tau}_{0}\right)$ and $1-\tilde{\pi}_{\tilde{\varphi}}\left(\tilde{\tau}_{1}\right)$ are called fuzzy type-I and fuzzy type-II errors denoted by $\widetilde{\alpha}_{\widetilde{\varphi}}$ and $\widetilde{\beta}_{\widetilde{\varphi}}$, respectively. 
Here, similar to the approaches introduced in Hesamian and Taheri (2013), Hesamian and Chachi (2013), the fuzzy $p$ value for testing hypotheses presented in Definition 5 is given as follows.

Definition 9 Consider testing the null hypothesis $\widetilde{H}_{0}: \tilde{\tau} \asymp$ $\tilde{\tau}_{0}$ versus the alternative one-sided hypothesis $\widetilde{H}_{1}: \tilde{\tau}(\succ) \prec$ $\tilde{\tau}_{0}$ or two-sided hypothesis $\widetilde{H}_{1}: \tilde{\tau} \neq \tilde{\tau}_{0}$. The fuzzy $p$ value is defined to be the fuzzy set $\widetilde{p}$ with $\alpha$-cuts $\left[\widetilde{p}_{\alpha}^{L}, \widetilde{p}_{\alpha}^{U}\right]$, where $\tilde{p}_{\alpha}^{L}=\inf _{\beta \geq \alpha} \inf \left\{\delta \in[0,1]: T\left(\widetilde{\mathbf{x}}_{\beta}\right) \in \mathbf{C}_{\delta}^{\beta}\right\}$,

and

$\widetilde{p}_{\alpha}^{U}=\sup _{\beta \geq \alpha} \inf \left\{\delta \in[0,1]: T\left(\widetilde{\mathbf{x}}_{\beta}\right) \in \mathbf{C}_{\delta}^{\beta}\right\}$,

where $T$ denotes the classical test statistic and $\mathbf{C}_{\delta}^{\beta}$ is the related critical region to test the null hypothesis $H_{0}^{\beta}: \widetilde{\tau}_{\beta}=$ $\left(\widetilde{\tau}_{0}\right)_{\beta}$ versus the alternative one-sided hypothesis $H_{1}^{\beta}: \widetilde{\tau}_{\beta}>$ $(<)\left(\widetilde{\tau}_{0}\right)_{\beta}$ (or two-sided hypothesis $H_{1}^{\beta}: \widetilde{\tau}_{\beta} \neq\left(\widetilde{\tau}_{0}\right)_{\beta}$ ) based on the random sample $\widetilde{\mathbf{X}}_{\beta}$.

Finally, in the problem of testing a hypothesis based on the fuzzy random sample $\widetilde{\mathbf{X}}=\left(\widetilde{X}_{1}, \ldots, \widetilde{X}_{n}\right)$, we propose the following definition of a fuzzy test function:

Definition 10 The fuzzy-valued function $\widetilde{\varphi}: \mathcal{F}^{n}(\mathbb{R}) \rightarrow$ $\mathcal{F}(\{0,1\})$ is called a fuzzy test whenever $\widetilde{\psi}(\widetilde{\mathbf{X}})$, as a fuzzy set on $\{0,1\}$, shows the degrees of rejection and acceptance of the hypothesis $H_{0}$ for any $\widetilde{\mathbf{X}}$. Here, "0" and " 1 " stand for acceptance and rejection of the null hypothesis, respectively, and $\mathcal{F}(\{0,1\})$ is the class of all fuzzy sets on $\{0,1\}$.

Definition 11 For the problem of testing hypotheses $\widetilde{H}_{0}$ : $\tilde{\tau} \asymp \widetilde{\tau}_{0}$ where $\widetilde{\tau}_{0} \in \mathcal{F}(\Theta)$, based on the fuzzy random sample $\widetilde{\mathbf{X}}=\left(\widetilde{X}_{1}, \ldots, \widetilde{X}_{n}\right)$ and at a given significance level $\delta \in$ $(0,1]$, the fuzzy test is defined to be the following fuzzy set

$\widetilde{\psi}(\widetilde{\mathbf{X}})=\left\{\frac{\operatorname{Cr}(\widetilde{p} \geq \delta)}{0}, \frac{\operatorname{Cr}(\widetilde{p}<\delta)}{1}\right\}$.

This test accepts the null hypothesis with the credibility degree of $\operatorname{Cr}(\widetilde{p} \geq \delta)$ (degree of acceptance), and rejects it with the credibility degree of $\operatorname{Cr}(\widetilde{p}<\delta)=1-\operatorname{Cr}(\widetilde{p} \geq \delta)$ (degree of rejection).

\subsection{Testing hypotheses about the fuzzy parameters of a normal FRV}

A particular class of parametric statistical testing hypotheses is the testing hypotheses about the parameters of a normal population. Here, we are going to apply the proposed method in the previous section for a normal FRV, i.e., assume that $\widetilde{\mathbf{X}}=\left(\widetilde{X}_{1}, \ldots, \widetilde{X}_{n}\right)$ be a random sample from a normal population and $\widetilde{\Theta}=\mathcal{F}(\Theta)$ be the class of all fuzzy numbers on the parameter space $\Theta=\left\{\left(\theta, \sigma^{2}\right): \theta \in \mathbb{R}, \sigma\right.$ $\in(0, \infty)\}$.

Remark 6 Consider testing $\widetilde{H}_{0}: \tilde{\theta} \asymp \tilde{\theta}_{0}$ for a normal FRV where $\widetilde{\sigma}^{2}$ is known. Therefore, the $\alpha$-cuts of the fuzzy $p$ value defined in Definition 9 are reduced as follows

1. for testing alternative hypothesis $\widetilde{H}_{1}: \tilde{\theta} \succ \tilde{\theta}_{0}$,

$$
\begin{aligned}
& \tilde{p}_{\alpha}^{L}=\inf _{\beta \geq \alpha}\left[1-\Phi\left(\frac{\sqrt{n}\left(\overline{\widetilde{x}}_{\beta}-\left(\tilde{\theta}_{0}\right)_{1-\beta}\right)}{\sqrt{\tilde{\sigma}_{1-\beta}^{2}}}\right)\right], \\
& \tilde{p}_{\alpha}^{U}=\sup _{\beta \geq \alpha}\left[1-\Phi\left(\frac{\sqrt{n}\left(\overline{\widetilde{x}}_{\beta}-\left(\widetilde{\theta}_{0}\right)_{1-\beta}\right)}{\sqrt{\widetilde{\sigma}_{1-\beta}^{2}}}\right)\right] .
\end{aligned}
$$

2. for testing alternative hypothesis $\widetilde{H}_{1}: \tilde{\theta} \prec \tilde{\theta}_{0}$,

$$
\begin{aligned}
& \tilde{p}_{\alpha}^{L}=\inf _{\beta \geq \alpha} \Phi\left(\frac{\sqrt{n}\left(\overline{\widetilde{x}}_{\beta}-\left(\widetilde{\theta}_{0}\right)_{1-\beta}\right)}{\sqrt{\widetilde{\sigma}_{1-\beta}^{2}}}\right), \\
& \tilde{p}_{\alpha}^{U}=\sup _{\beta \geq \alpha} \Phi\left(\frac{\sqrt{n}\left(\overline{\widetilde{x}}_{1-\beta}-\left(\widetilde{\theta}_{0}\right)_{1-\beta}\right)}{\sqrt{\widetilde{\sigma}_{1-\beta}^{2}}}\right) .
\end{aligned}
$$

3. for testing alternative hypothesis $\widetilde{H}_{1}: \tilde{\theta} \neq \tilde{\theta}_{0}$,

$$
\begin{aligned}
& \tilde{p}_{\alpha}^{L}=2 \inf _{\beta \geq \alpha} \Phi\left(-\sqrt{n}\left|\frac{\overline{\widetilde{x}}_{\beta}-\left(\tilde{\theta}_{0}\right)_{1-\beta}}{\sqrt{\widetilde{\sigma}_{1-\beta}^{2}}}\right|\right), \\
& \widetilde{p}_{\alpha}^{U}=2 \sup _{\beta \geq \alpha} \Phi\left(-\sqrt{n}\left|\frac{\widetilde{\widetilde{x}}_{\beta}-\left(\widetilde{\theta}_{0}\right)_{1-\beta}}{\sqrt{\widetilde{\sigma}_{1-\beta}^{2}}}\right|\right),
\end{aligned}
$$

where $\Phi$ denotes the standard normal cumulative distribution function and $\overline{\widetilde{x}}_{\beta}=1 / n \sum_{i=1}^{n}\left(\widetilde{x}_{i}\right)_{\beta}$.

Now assume that $\widetilde{\sigma}^{2}$ is unknown. In this case, the $\alpha$-cuts of the fuzzy $p$ value are given as follows

1. for testing alternative hypothesis $\widetilde{H}_{1}: \tilde{\theta} \succ \tilde{\theta}_{0}$,

$$
\begin{aligned}
& \tilde{p}_{\alpha}^{L}=\inf _{\beta \geq \alpha} \mathbf{P}\left(\mathbf{t}_{n-1} \geq \frac{\sqrt{n}\left(\overline{\widetilde{x}}_{\beta}-\left(\widetilde{\theta}_{0}\right)_{1-\beta}\right)}{\sqrt{\widetilde{s}_{1-\beta}^{2}}}\right), \\
& \tilde{p}_{\alpha}^{U}=\sup _{\beta \geq \alpha} \mathbf{P}\left(\mathbf{t}_{n-1} \geq \frac{\sqrt{n}\left(\overline{\widetilde{x}}_{\beta}-\left(\widetilde{\theta}_{0}\right)_{1-\beta}\right)}{\sqrt{\tilde{s}_{1-\beta}^{2}}}\right) .
\end{aligned}
$$


2. for testing alternative hypothesis $\widetilde{H}_{1}: \tilde{\theta} \prec \tilde{\theta}_{0}$,

$$
\begin{aligned}
& \tilde{p}_{\alpha}^{L}=\inf _{\beta \geq \alpha} \mathbf{P}\left(\mathbf{t}_{n-1} \leq \frac{\sqrt{n}\left(\overline{\widetilde{x}}_{\beta}-\left(\widetilde{\theta}_{0}\right)_{1-\beta}\right)}{\sqrt{\tilde{s}_{1-\beta}^{2}}}\right), \\
& \tilde{p}_{\alpha}^{U}=\sup _{\beta \geq \alpha} \mathbf{P}\left(\mathbf{t}_{n-1} \leq \frac{\sqrt{n}\left(\overline{\widetilde{x}}_{\beta}-\left(\widetilde{\theta}_{0}\right)_{1-\beta}\right)}{\sqrt{\tilde{s}_{1-\beta}^{2}}}\right) .
\end{aligned}
$$

3. for testing alternative hypothesis $\widetilde{H}_{1}: \tilde{\theta} \neq \tilde{\theta}_{0}$,

$$
\begin{aligned}
& \tilde{p}_{\alpha}^{L}=2 \inf _{\beta \geq \alpha} \mathbf{P}\left(\mathbf{t}_{n-1} \leq-\sqrt{n}\left|\frac{\overline{\widetilde{x}}_{\beta}-\left(\widetilde{\theta}_{0}\right)_{1-\beta}}{\sqrt{\widetilde{s}_{1-\beta}^{2}}}\right|\right), \\
& \widetilde{p}_{\alpha}^{U}=2 \sup _{\beta \geq \alpha} \mathbf{P}\left(\mathbf{t}_{n-1} \leq-\sqrt{n}\left|\frac{\overline{\widetilde{x}}_{\beta}-\left(\widetilde{\theta}_{0}\right)_{1-\beta}}{\sqrt{\widetilde{s}_{1-\beta}^{2}}}\right|\right),
\end{aligned}
$$

where $\mathbf{t}_{n-1}$ denotes the $\mathbf{t}$-student distribution with degree of freedom $n-1$ and $\widetilde{s}_{\beta}^{2}=\frac{1}{n-1} \sum_{i=1}^{n}\left(\left(\widetilde{x}_{i}\right)_{\beta}-\overline{\widetilde{x}}_{\beta}\right)^{2}$.

Remark 7 Here, we describe calculating the fuzzy power to test about $\widetilde{\mu}$ for a normal FRV. We explain the method for the case of testing $\widetilde{H}_{0}: \tilde{\theta}=\tilde{\theta}_{0}$ versus $\widetilde{H}_{1}: \tilde{\theta} \succ \tilde{\theta}_{0}$ (the other cases can be obtained similarly). First, assume that $\tilde{\sigma}^{2}$ is known. From Definition 7, it is easy to verify that the $\alpha$-cuts of the fuzzy power at $\widetilde{\theta}^{*}\left(\succ \tilde{\theta}_{0}\right)$ are obtain as follows

$$
\begin{aligned}
& \left(\widetilde{\pi}_{\tilde{\varphi}}\left(\widetilde{\theta}^{*}\right)\right)_{\alpha}^{L}=\inf _{\beta \geq \alpha}\left[1-\Phi\left(\sqrt{n} \frac{\left(c_{\beta}-\left(\widetilde{\theta}^{*}\right)_{1-\beta}\right)}{\sqrt{\tilde{\sigma}_{1-\beta}^{2}}}\right)\right], \\
& \left(\widetilde{\pi}_{\tilde{\varphi}}\left(\widetilde{\theta}^{*}\right)\right)_{\alpha}^{U}=\sup _{\beta \geq \alpha}\left[1-\Phi\left(\sqrt{n} \frac{\left(c_{\beta}-\left(\widetilde{\theta}^{*}\right)_{1-\beta}\right)}{\sqrt{\widetilde{\sigma}_{1-\beta}^{2}}}\right)\right],
\end{aligned}
$$

where $c_{\beta}=\left(\widetilde{\theta}_{0}\right)_{1-\beta}+z_{1-\frac{\delta}{2}} \frac{\sqrt{\widetilde{\sigma}_{1-\beta}^{2}}}{\sqrt{n}}$.

Now if $\widetilde{\sigma}^{2}$ is unknown then, for testing $\widetilde{H}_{0}: \tilde{\theta}=\tilde{\theta}_{0}$ versus $\widetilde{H}_{1}: \tilde{\theta} \succ \tilde{\theta}_{0}$, the $\alpha$-cuts of $\widetilde{\pi}_{\tilde{\varphi}}$ at $\widetilde{\theta}^{*}\left(\succ \tilde{\theta}_{0}\right)$ are given by

$\left(\widetilde{\pi}_{\tilde{\varphi}}\left(\widetilde{\theta}^{*}\right)\right)_{\alpha}^{L}=\inf _{\beta \geq \alpha}\left[1-\mathbf{P}\left(\mathbf{t}_{n-1} \leq \sqrt{n} \frac{\left(c_{\beta}-\left(\widetilde{\theta}^{*}\right)_{1-\beta}\right)}{\sqrt{\widetilde{s}_{1-\beta}^{2}}}\right)\right]$,

$\left(\tilde{\pi}_{\varphi}\left(\widetilde{\theta}^{*}\right)\right)_{\alpha}^{U}=\sup _{\beta \geq \alpha}\left[1-\mathbf{P}\left(\mathbf{t}_{n-1} \leq \sqrt{n} \frac{\left(c_{\beta}-\left(\widetilde{\theta}^{*}\right)_{1-\beta}\right)}{\sqrt{\widetilde{s}_{1-\beta}^{2}}}\right)\right]$,

where $c_{\beta}=\left(\widetilde{\theta}_{0}\right)_{1-\beta}+\mathbf{t}_{n-1, \frac{\delta}{2}} \frac{\sqrt{\tilde{s}_{1-\beta}^{2}}}{\sqrt{n}}$.

Remark 8 Now, consider testing the null hypothesis $\widetilde{H}_{0}$ : $\tilde{\sigma}^{2} \asymp \tilde{\sigma}_{0}^{2}$. From Definition 9 , the $\alpha$-cuts of the fuzzy $p$ value are given by $\left[\widetilde{p}_{\alpha}^{L}, \widetilde{p}_{\alpha}^{U}\right]$, where
1. for testing alternative hypothesis $\widetilde{H}_{1}: \tilde{\sigma}^{2} \succ \tilde{\sigma}_{0}^{2}$,

$$
\begin{aligned}
\widetilde{p}_{\alpha}^{L} & =\inf _{\beta \geq \alpha} \mathbf{P}_{H_{0}^{\beta}: \widetilde{\sigma}_{\beta}^{2}=\left(\widetilde{\sigma}_{0}^{2}\right)_{\beta}}\left(\widetilde{S}_{\beta}^{2} \geq \widetilde{s}_{\beta}^{2}\right) \\
& =\inf _{\beta \geq \alpha} \mathbf{P}\left(\chi_{n-1}^{2} \geq \frac{(n-1) \widetilde{s}_{\beta}^{2}}{\left(\widetilde{\sigma}_{0}^{2}\right)_{1-\beta}}\right), \\
\tilde{p}_{\alpha}^{U} & =\sup _{\beta \geq \alpha} \mathbf{P}_{H_{0}^{\beta}: \widetilde{\sigma}_{\beta}^{2}=\left(\widetilde{\sigma}_{0}^{2}\right)_{\beta}}\left(\widetilde{S}_{\beta}^{2} \geq \widetilde{s}_{\beta}^{2}\right), \\
& =\sup _{\beta \geq \alpha} \mathbf{P}\left(\chi_{n-1}^{2} \geq \frac{(n-1) \widetilde{s}_{\beta}^{2}}{\left(\widetilde{\sigma}_{0}^{2}\right)_{1-\beta}}\right) .
\end{aligned}
$$

2. for testing alternative hypothesis $\widetilde{H}_{1}: \tilde{\sigma}^{2} \prec \tilde{\sigma}_{0}^{2}$,

$$
\begin{aligned}
\widetilde{p}_{\alpha}^{L} & =\inf _{\beta \geq \alpha} \mathbf{P}_{H_{0}^{\beta}: \tilde{\sigma}_{\beta}^{2}=\left(\widetilde{\sigma}_{0}^{2}\right)_{\beta}}\left(\widetilde{S}_{\beta}^{2} \leq \widetilde{s}_{\beta}^{2}\right), \\
& =\inf _{\beta \geq \alpha} \mathbf{P}\left(\chi_{n-1}^{2} \leq \frac{(n-1) \widetilde{s}_{\beta}^{2}}{\left(\widetilde{\sigma}_{0}^{2}\right)_{1-\beta}}\right), \\
\widetilde{p}_{\alpha}^{U} & =\sup _{\beta \geq \alpha} \mathbf{P}_{H_{0}^{\beta}: \tilde{\sigma}_{\beta}^{2}=\left(\widetilde{\sigma}_{0}^{2}\right)_{\beta}}\left(\widetilde{S}_{\beta}^{2} \leq \widetilde{s}_{\beta}^{2}\right), \\
& =\sup _{\beta \geq \alpha} \mathbf{P}\left(\chi_{n-1}^{2} \leq \frac{(n-1) \widetilde{s}_{\beta}^{2}}{\left(\widetilde{\sigma}_{0}^{2}\right)_{1-\beta}}\right) .
\end{aligned}
$$

3. for testing alternative hypothesis $\widetilde{H}_{1}: \tilde{\sigma}^{2} \neq \tilde{\sigma}_{0}^{2}$,

$$
\begin{aligned}
& \tilde{p}_{\alpha}^{L}=2 \inf _{\beta \geq \alpha} \min \left\{\mathbf{P}_{H_{0}^{\beta}: \widetilde{\sigma}_{\beta}^{2}=\left(\widetilde{\sigma}_{0}^{2}\right)_{\beta}}\left(\widetilde{S}_{\beta}^{2} \leq \widetilde{s}_{\beta}^{2}\right),\right. \\
& \left.\mathbf{P}_{H_{0}^{\beta}: \widetilde{\sigma}_{\beta}^{2}=\left(\widetilde{\sigma}_{0}^{2}\right)_{\beta}}\left(\widetilde{S}_{\beta}^{2} \geq \widetilde{s}_{\beta}^{2}\right)\right\}, \\
& =2 \inf _{\beta \geq \alpha} \min \left\{\mathbf{P}\left(\chi_{n-1}^{2} \leq \frac{(n-1) \widetilde{s}_{\beta}^{2}}{\left(\widetilde{\sigma}_{0}^{2}\right)_{1-\beta}}\right)\right. \text {, } \\
& \left.\mathbf{P}\left(\chi_{n-1}^{2} \geq \frac{(n-1) \widetilde{s}_{\beta}^{2}}{\left(\widetilde{\sigma}_{0}^{2}\right)_{1-\beta}}\right)\right\}, \\
& \tilde{p}_{\alpha}^{U}=2 \sup _{\beta \geq \alpha} \min \left\{\mathbf{P}_{H_{0}^{\beta}: \widetilde{\sigma}_{\beta}^{2}=\left(\widetilde{\sigma}_{0}^{2}\right)_{\beta}}\left(\widetilde{S}_{\beta}^{2} \leq \widetilde{s}_{\beta}^{2}\right)\right. \text {, } \\
& \left.\mathbf{P}_{H_{0}^{\beta}: \widetilde{\sigma}_{\beta}^{2}=\left(\widetilde{\sigma}_{0}^{2}\right)_{\beta}}\left(\widetilde{S}_{\beta}^{2} \geq \widetilde{s}_{\beta}^{2}\right)\right\}, \\
& =2 \sup _{\beta \geq \alpha} \min \left\{\mathbf{P}\left(\chi_{n-1}^{2} \leq \frac{(n-1) \widetilde{s}_{\beta}^{2}}{\left(\widetilde{\sigma}_{0}^{2}\right)_{1-\beta}}\right)\right. \text {, } \\
& \left.\mathbf{P}\left(\chi_{n-1}^{2} \geq \frac{(n-1) \widetilde{s}_{\beta}^{2}}{\left(\widetilde{\sigma}_{0}^{2}\right)_{1-\beta}}\right)\right\},
\end{aligned}
$$

where $\chi_{n-1}^{2}$ denotes the Chi-square distribution with degree of freedom $n-1$. 
By a similar manner described by Remarks $6-8$, we can obtain the fuzzy power and fuzzy $p$ value for testing about $\widetilde{\sigma^{2}}$ for a normal FRV.

Remark 9 It should be mentioned that, based on Remark 5, all definitions of hypotheses given in this paper are just a rewritten version of Wu's ones ( Wu 2005).

\section{Numerical example}

In this section, we provide a numerical example to clarify the discussions in this paper and give the possible application of the proposed method for normal FRVs.

Example 2 (Elsherif et al. 2009) Based on a random sample of the received signal from a target, assume we have fuzzy observations $\widetilde{\mathbf{x}}=\left(\widetilde{x}_{1}, \widetilde{x}_{2}, \ldots, \widetilde{x}_{25}\right)$ (as shown in Table 1) measured in nanowatt which is approximately normally distributed $N\left(\widetilde{\theta}, \widetilde{\sigma}^{2}\right)$. We wish to test the following hypothesis:

$\left\{\begin{array}{l}\widetilde{H}_{0}: \tilde{\theta} \asymp \tilde{\theta}_{0} \asymp(1.35,1.50,1.80)_{T}, \\ \widetilde{H}_{1}: \tilde{\theta} \prec \widetilde{\theta}_{0} .\end{array}\right.$

Applying the procedure proposed in Sect. 4.1, using Remark 6, the fuzzy $p$ value is derived point by point and its membership function is shown in Fig. 1. This membership function can be interpreted as "about 0.075 ". Therefore, at level of $\delta=0.05$, the fuzzy test for testing $\widetilde{H}_{0}: \tilde{\theta} \asymp$ $(1.35,1.50,1.80)_{T}$ is obtained as $\widetilde{\psi}(\widetilde{\mathbf{X}})=\left\{\frac{0.905}{0}, \frac{0.095}{1}\right\}$. So, with respect to the observed fuzzy observations and at significance level of $\delta=0.05$, the null hypothesis $\widetilde{H}_{0}: \tilde{\theta} \asymp$ $(1.35,1.50,1.80)_{T}$ is accepted with a degree of 0.905 and it is rejected with a degree of 0.095 . Such a result may be interpreted as "we absolutely tend to accept $\widetilde{H}_{0}$ ". In addition, based on Remark 7, the membership of the fuzzy power at $\tilde{\theta}^{*} \asymp(0.50,0.70,0.80)_{T}$ is shown in Fig. 2 which is interpreted as "about 0.999 ".

Now, suppose that we want to test the following hypotheses about $\widetilde{\sigma^{2}}$ :

$$
\left\{\begin{array}{l}
\widetilde{H}_{0}: \widetilde{\sigma}^{2} \asymp \widetilde{\sigma}_{0}^{2} \asymp(0.30,0.50,0.70)_{T}, \\
\widetilde{H}_{1}: \widetilde{\sigma}^{2} \succ \widetilde{\sigma}_{0}^{2} .
\end{array}\right.
$$

From Remark 6, the membership function of the fuzzy $p$ value is obtained as "about 0.83 " whose membership function is shown in Fig. 3. Therefore, the fuzzy test to test $\widetilde{H}_{0}: \widetilde{\sigma}^{2} \asymp$ $(0.30,0.50,0.70)_{T}$ versus $\widetilde{H}_{1}: \widetilde{\sigma}^{2} \succ \widetilde{\sigma}_{0}^{2}$, at significance level of $\delta=0.05$, is obtained as $\widetilde{\psi}(\widetilde{\mathbf{X}})=\left\{\frac{0.991}{0}, \frac{0.009}{1}\right\}$. In such a case, therefore, we are strongly convinced to accept
Table 1 The observed fuzzy random sample in Example 2

\begin{tabular}{lll}
\hline$\widetilde{x}_{1}=(0.02,0.10,0.23)_{T}$ & $\widetilde{x}_{2}=(0.08,0.20,0.37)_{T}$ & $\widetilde{x}_{3}=(0.15,0.30,0.46)_{T}$ \\
$\widetilde{x}_{4}=(0.28,0.40,0.56)_{T}$ & $\widetilde{x}_{5}=(0.37,0.50,0.65)_{T}$ & $\widetilde{x}_{6}=(0.42,0.60,0.74)_{T}$ \\
$\widetilde{x}_{7}=(0.54,0.70,0.83)_{T}$ & $\widetilde{x}_{8}=(0.66,0.80,0.93)_{T}$ & $\widetilde{x}_{9}=(0.72,0.90,1.26)_{T}$ \\
$\widetilde{x}_{10}=(0.85,1.00,1.15)_{T}$ & $\widetilde{x}_{11}=(0.98,1.10,1.22)_{T}$ & $\widetilde{x}_{12}=(1.05,1.20,1.32)_{T}$ \\
$\widetilde{x}_{13}=(1.17,1.30,1.48)_{T}$ & $\widetilde{x}_{14}=(1.25,1.40,1.54)_{T}$ & $\widetilde{x}_{15}=(1.32,1.50,1.67)_{T}$ \\
$\widetilde{x}_{16}=(1.37,1.60,1.78)_{T}$ & $\widetilde{x}_{17}=(1.53,1.70,1.8)_{T}$ & $\widetilde{x}_{18}=(1.62,1.80,1.97)_{T}$ \\
$\widetilde{x}_{19}=(1.75,1.90,2.08)_{T}$ & $\widetilde{x}_{20}=(1.76,2.00,2.23)_{T}$ & $\widetilde{x}_{21}=(1.93,2.10,2.27)_{T}$ \\
$\widetilde{x}_{22}=(2.06,2.20,2.35)_{T}$ & $\widetilde{x}_{23}=(2.05,2.30,2.46)_{T}$ & $\widetilde{x}_{24}=(2.21,2.40,2.55)_{T}$ \\
$\widetilde{x}_{25}=(2.32,2.50,2.60)_{T}$ & - & - \\
\hline
\end{tabular}

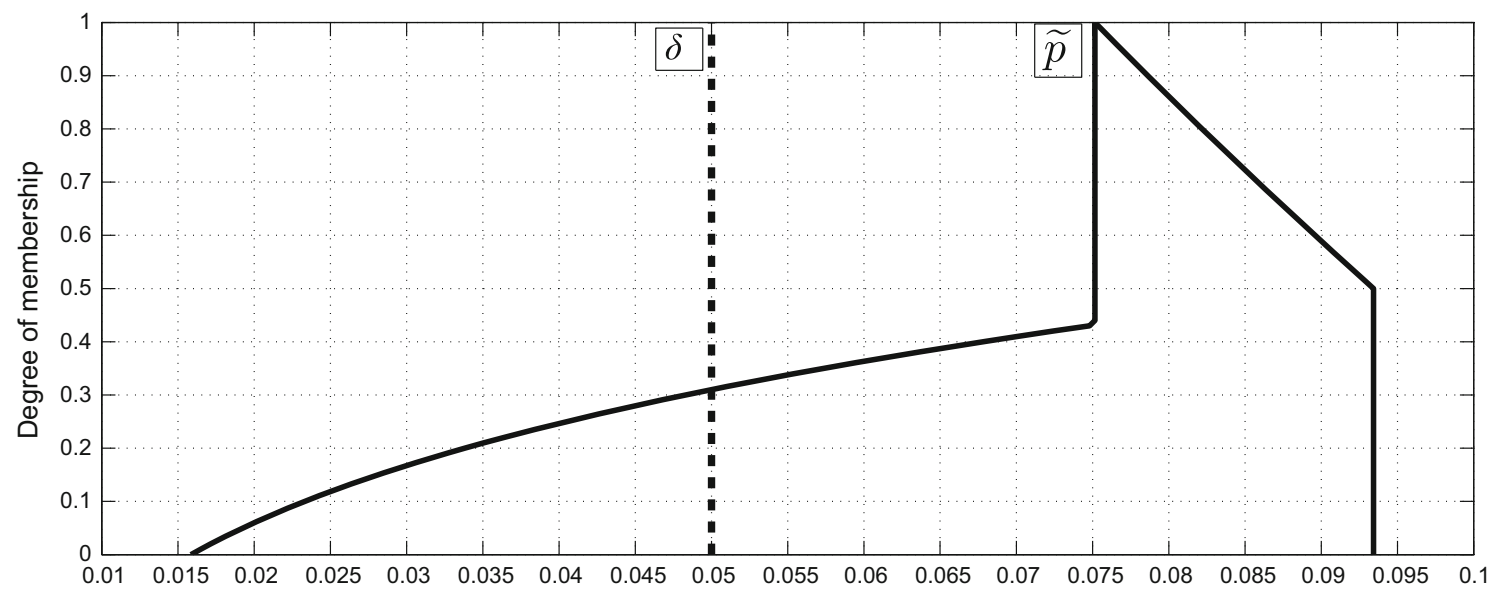

Fig. 1 The membership function of the fuzzy $p$ value and the significance level in Example 2 for testing $\widetilde{H}_{0}: \tilde{\theta} \asymp(1.35,1.50,1.80)_{T}$ against $\widetilde{H}_{1}: \tilde{\theta} \prec(1.35,1.50,1.80)_{T}$ 


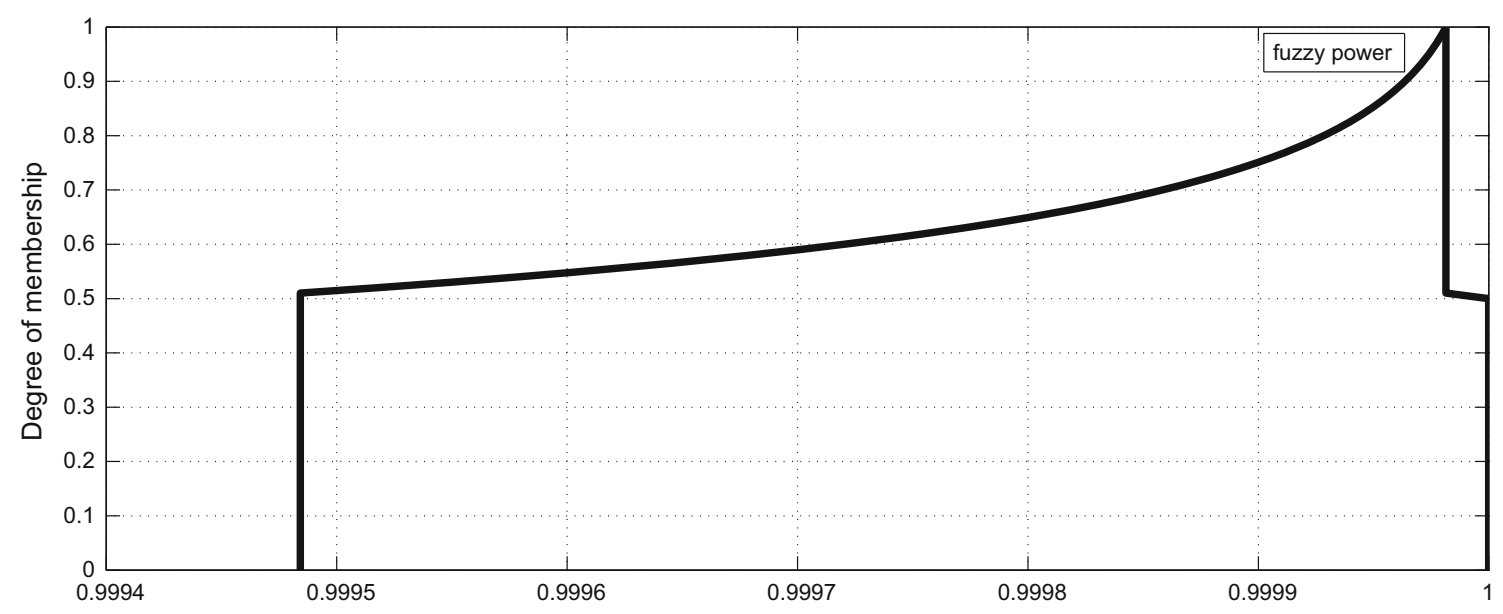

Fig. 2 The membership function of the fuzzy power at $\tilde{\theta}^{*} \asymp(0.50,0.70,0.80)_{T}$ in Example 2

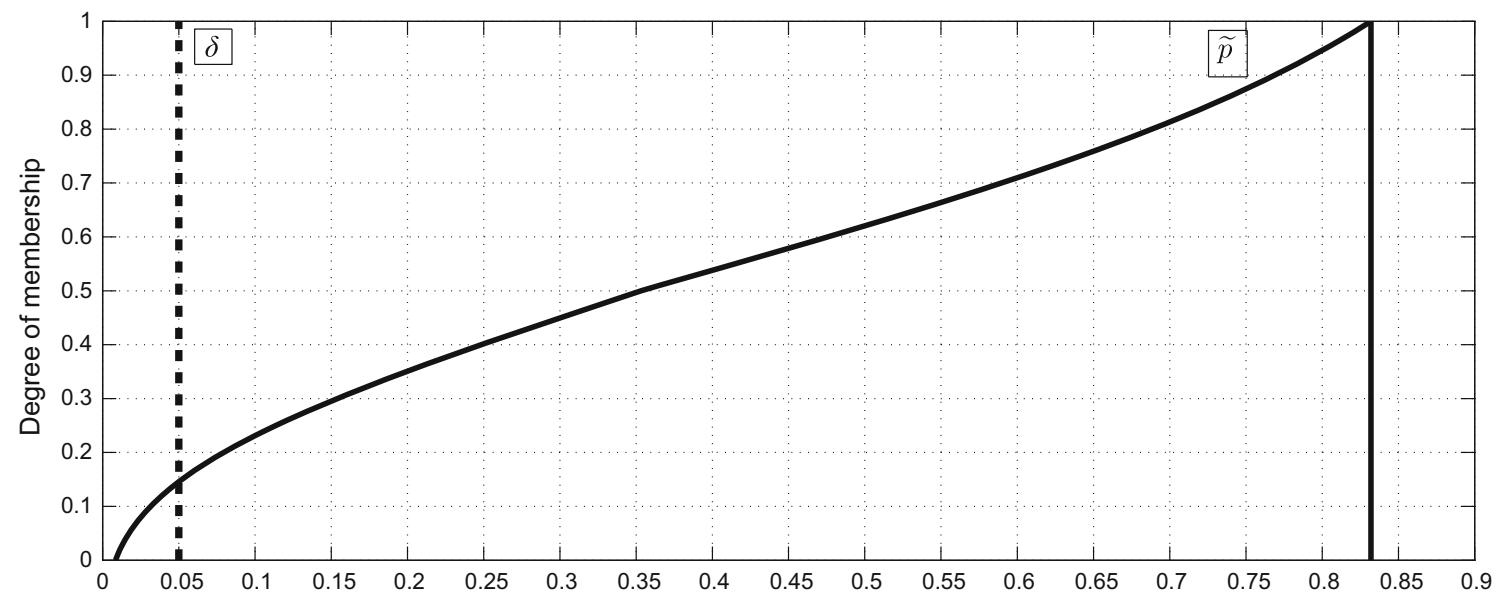

Fig. 3 The membership function of the fuzzy $p$ value and the significance level in Example 2 for testing $\widetilde{H}_{0}: \widetilde{\sigma}^{2} \asymp(0.30,0.50,0.70)_{T}$ against $\widetilde{H}_{1}: \widetilde{\sigma}^{2} \succ(0.30,0.50,0.70)_{T}$

$\widetilde{H}_{0}: \widetilde{\sigma}^{2} \asymp(0.30,0.50,0.70)_{T}$, at significance level of $\delta=$ 0.05 .

\section{A comparison study}

It should be mentioned that Akbari and Rezaei (2009, 2010) extended the classical approach for testing statistical hypotheses about the (crisp) mean or variance of a normal distribution by introducing some fuzzy numbers to interpret the sentences "larger than", "smaller than" or "not equal" for population's parameters based on imprecise observations. Parchami et al. (2010) defined an extension of fuzzy $p$ value for a crisp random sample of a normal distribution to test imprecisely hypothesis test about the mean. Arefi and Taheri (2011) extended the classical critical region for testing fuzzy hypothesis about the parameters of the classical normal distribution based on fuzzy data. Wu (2005) considered the problem of hypotheses test with fuzzy data. Based on a concept of fuzzy random variable, first he defined the fuzzy mean of the normal distribution. Then, based on a proposed ranking method, he defined the hypotheses about the population's fuzzy mean. He considered two cases: (1) the variance of the population is known, (2) the variance of the population is unknown. Finally, he introduced a fuzzy test based on the classical critical region. However, for both cases, he assigned a crisp number into the structure of testing hypothesis's method: for the case (1) the variance of the population is considered as a crisp number and for the case (2) the variance of the fuzzy sample is calculated based on core of the fuzzy data as a crisp number. As we observe, in all above methods, the variance of the normal population is considered as a crisp number. However, for testing fuzzy hypothesis about mean of a normal population, it is reasonable that the variance of the population is also considered as a imprecise value. So, it may be an advantage of our method with respect to abovementioned methods. Geyer and Meeden considered the problem of the classical optimal hypothesis tests for the binomial 
distribution (in general for discrete data with crisp parameter) using an interval estimation of a binomial proportion (Geyer and Meeden 2005). They introduced the notion of fuzzy confidence intervals by inverting families of randomized tests. Then, they introduce a notion of fuzzy $p$ value in which it is only a function of the parameters of the model. Finally, they provided a unified description of fuzzy confidence interval, fuzzy $p$ values and fuzzy decision. However, their proposed method is not a fuzzy method in general, since this approach does not consider the imprecise information about the model such as imprecise observations or imprecise hypotheses and it does not lead to a fuzzy decision. Viertl (2011) and Filzmoser and Viertl (2004) also extend a concept of fuzzy $p$ value when the observations are fuzzy and hypotheses are crisp. Arnold $(1996,1998)$ proposed a method for testing fuzzy hypotheses about the population parameter with crisp data. He provided some definitions for the probability of type-I and type-II errors and presented the best test for the oneparameter exponential family.

As it is observed, in all above-proposed methods for testing statistical hypothesis, at least one of the essential population's information such as data, hypothesis or population's parameters play a crisp role in the structure of testing procedure. While, by restructuring the concept of fuzzy random variable, we involved the population's information including: fuzzy data and fuzzy parameters into the hypothesis test, type-I error (or type-II error) and power of test. Finally, we obtained a fuzzy test to make decision for accepting or rejecting the fuzzy hypothesis of interest about the fuzzy parameters.

\section{Conclusions}

This paper proposes a method for testing hypotheses about the fuzzy parameters of a fuzzy random variable. In this approach, we reconstruct a well-known concept of a fuzzy random variable using the concept of $\alpha$-optimistic values. Then, we extended the concepts of the fuzzy power test and fuzzy $p$ value. Finally, based on the credibility index, to compare the observed fuzzy $p$ value and the crisp significance level, the fuzzy hypothesis of interest can be accepted or rejected with degrees of conviction between 0 and 1.

Although we focused on testing hypotheses about the parameters of a normal fuzzy random variable, the proposed method is general and it can be applied for other kinds of fuzzy random variables as well. Moreover, it can be applied for other kind of testing hypothesis such as comparing two independent fuzzy random variables of two populations, the one-way or two-way analysis of variance and nonparametric approaches including testing hypothesis. However, the proposed method can be applied only for fuzzy numbers involved in a problem of statistical hypothesis testing. Moreover, the topic of testing hypothesis for fuzzy parameters of a fuzzy random variable can be extended to the case where the level of significance is given by a fuzzy number, too. To do this, one can easily apply the credibility index for comparing two fuzzy numbers into the decision making.

Open Access This article is distributed under the terms of the Creative Commons Attribution License which permits any use, distribution, and reproduction in any medium, provided the original author(s) and the source are credited.

\section{Appendix: Proof of the main results}

This appendix provides the mathematical proofs of the theoretical results in our paper.

Proof of Theorem 1 (i) Let $g_{\widetilde{A}}: \mathbb{R} \rightarrow[0,1]$ be given by $g_{\widetilde{A}}(x)=\operatorname{Cr}(\widetilde{A} \geq x)$. Then

$\widetilde{A}_{\alpha}=\sup \left\{x \in \widetilde{A}[0] \mid g_{\widetilde{A}}(x) \geq \alpha\right\}$.

First, let $\alpha \in[0,0.5)$. Since

$$
\begin{aligned}
g_{\widetilde{A}}\left(\widetilde{A}_{2 \alpha}^{U}\right) & =\frac{1}{2}\left(\sup _{y \in\left[\widetilde{A}_{2 \alpha}^{U},+\infty\right)} \mu_{\widetilde{A}}(y)+1 \sup _{\left(-\infty, \widetilde{A}_{2 \alpha}^{U}\right)} \mu_{\widetilde{A}}(y)\right) \\
& =\frac{1}{2}(2 \alpha)=\alpha,
\end{aligned}
$$

we have $\widetilde{A}_{\alpha} \geq \widetilde{A}_{2 \alpha}^{U}$. On the other hand, for each $z>\widetilde{A}_{2 \alpha}^{U}$, $\sup _{y \in[z,+\infty)} \mu_{\widetilde{A}}(y)=\widetilde{A}(z)$ and $\sup _{y \in(-\infty, z)} \mu_{\widetilde{A}}(y)=$ 1. Therefore,

$$
\begin{aligned}
g_{\widetilde{A}}(z) & =\operatorname{Cr}(\widetilde{A} \geq z) \\
& =\frac{1}{2}\left(\sup _{y \in[z,+\infty)} \mu_{\widetilde{A}}(y)+1-\sup _{y \in(-\infty, z)} \mu_{\widetilde{A}}(y)\right) \\
& =\frac{1}{2} \widetilde{A}(z)<\alpha .
\end{aligned}
$$

Hence, $\left\{x \in \mathbb{R} \mid g_{\widetilde{A}}(x) \geq \alpha\right\} \cap\left(\widetilde{A}_{2 \alpha}^{U},+\infty\right)=\emptyset$, which by the previous part implies that $\widetilde{A}_{\alpha}=\widetilde{A}_{2 \alpha}^{U}$.

Now suppose $\alpha \in[0.5,1]$ and let $x_{0}:=\sup \left\{x \leq x^{*} \mid\right.$ $\left.\mu_{\widetilde{A}}(x) \leq 2(1-\alpha)\right\}$. Then, $\mu_{\widetilde{A}}(x) \leq 2(1-\alpha)$, for all $x<x_{0}$. Hence,

$\sup _{y \in\left[x_{0},+\infty\right)} \mu_{\widetilde{A}}(y)=1$ and $\sup _{y \in\left(-\infty, x_{0}\right)} \mu_{\widetilde{A}}(y) \leq 2(1-\alpha)$.

Therefore, $g_{\widetilde{A}}\left(x_{0}\right) \geq \alpha$ which implies that $\phi_{\widetilde{A}}(\alpha) \geq x_{0}$. On the other hand, for $x>x_{0}$, since $\mu_{\widetilde{A}}(x)>2(1-\alpha)$, 
we have

$$
\begin{aligned}
g_{\widetilde{A}}(x) & =\frac{1}{2}\left(\sup _{y \in[x,+\infty)} \mu_{\widetilde{A}}(y)+1-\sup _{y \in(-\infty, x)} \mu_{\widetilde{A}}(y)\right) \\
& \geq \frac{1}{2}\left(1+1-\mu_{\widetilde{A}}(x)\right)<\alpha .
\end{aligned}
$$

Hence, $\phi_{\widetilde{A}}(\alpha)=x_{0}$. Note that, according to what has been proved, $\phi_{\widetilde{A}}(0.5)=\sup \left\{x \leq x^{*} \mid \mu_{\widetilde{A}}(x) \leq 1\right\}=$ $x^{*}=\widetilde{A}_{2(0.5)}^{U}$. Thus, the law of $\phi_{\widetilde{A}}$ can be written in the following form.

$$
\begin{aligned}
\forall \alpha & \in[0,1], \quad \phi_{\widetilde{A}}(\alpha) \\
& = \begin{cases}\widetilde{A}_{2 \alpha}^{U} & 0 \leq \alpha \leq 0.5, \\
\sup \left\{x \leq x^{*} \mid \mu_{\widetilde{A}}(x) \leq 2(1-\alpha)\right\} & 0.5 \leq \alpha \leq 1 .\end{cases}
\end{aligned}
$$

(ii) It is clear that $\phi_{\widetilde{A}}$ is decreasing on $[0,1]$. By the previous part, since for each $\alpha \in[0,0.5], \phi_{\widetilde{A}}(\alpha)=\widetilde{A}_{2 \alpha}^{U}$, using Lemma $1, \phi_{\widetilde{A}}$ is left continuous on $[0,0.5]$.

For $\alpha \in(0.5,1]$, suppose $\left\{\alpha_{n}\right\}_{n \in \mathbb{N}}$ is an increasing sequence in $(0.5,1]$ with $\alpha_{n} \rightarrow \alpha$. First, suppose that $\phi_{\widetilde{A}}(\alpha)=x^{*}$. Then, for each $x<x^{*}, \mu_{\widetilde{A}}(x) \leq 2(1-\alpha) \leq$ $2\left(1-\alpha_{n}\right)$. Hence $\phi_{\widetilde{A}}\left(\alpha_{n}\right) \geq x^{*}$, for all $n \in \mathbb{N}$. On the other hand, since $\alpha_{n}>0.5$, we have $\phi_{\widetilde{A}}\left(\alpha_{n}\right) \leq x^{*}$. Combining the two arguments, we obtain $\phi_{\widetilde{A}}\left(\alpha_{n}\right)=x^{*}$, for all $n \in \mathbb{N}$. Hence, in this case $\phi_{\widetilde{A}}\left(\alpha_{n}\right)=x^{*} \rightarrow \phi_{\widetilde{A}}(\alpha)=x^{*}$. Now suppose $\left.\phi_{\widetilde{A}^{\alpha}} \alpha\right)<x^{*}$. Then, for each $y \in \mathbb{R}$ with $\phi_{\widetilde{A}}(\alpha)<y<x^{*}$, $\mu_{\widetilde{A}}(y)>2(1-\alpha)$. Therefore, $\mu_{\widetilde{A}}(y)>2\left(1-\alpha_{n}\right)$, for large values of $n \in \mathbb{N}$. This implies that $\phi_{\widetilde{A}}\left(\alpha_{n}\right) \leq y$. Hence $\phi_{\widetilde{A}}\left(\alpha_{n}\right) \rightarrow \phi_{\widetilde{A}}(\alpha)$. This completes the proof of the left continuity of $\phi_{\widetilde{A}}$ on $(0.5,1]$.

Proof of Lemma 2 First suppose $\alpha_{0} \in(0.5,1]$ is a point of continuity of $\psi_{\widetilde{A}}$. Let $x_{0}:=\phi_{\widetilde{A}}\left(\alpha_{0}\right)$. Then $\widetilde{A}_{2\left(1-\alpha_{0}\right)}^{L} \leq x_{0}$. If $\widetilde{A}_{2\left(1-\alpha_{0}\right)}^{L}<x_{0}$ then $\mu_{\widetilde{A}}(x)=2\left(1-\alpha_{0}\right)$, for all $x \in$ $\left[\widetilde{A}_{2\left(1-\alpha_{0}\right)}^{L}, x_{0}\right)$. In this case for each $\alpha<\alpha_{0}$, since $2(1-\alpha)>$ $2\left(1-\alpha_{0}\right)$, we have $\widetilde{A}_{2(1-\alpha)}^{L} \geq x_{0}$ which contradicts the assumption of continuity of $\psi_{\widetilde{A}}$ at $\alpha_{0}$. Hence

$\psi_{\widetilde{A}}\left(\alpha_{0}\right)=\widetilde{A}_{2\left(1-\alpha_{0}\right)}^{L}=x_{0}=\phi_{\widetilde{A}}\left(\alpha_{0}\right)$.

Suppose, on the contrary, that $\alpha_{0}$ is a point of discontinuity of $\psi_{\widetilde{A}}$. Then, there $\epsilon_{0}>0$ such that for each $n \in \mathbb{N}$, one can find $\alpha_{n} \in\left(\alpha_{0}-\frac{1}{n}, \alpha\right)$ with $\psi_{\widetilde{A}}\left(\alpha_{n}\right)>\psi_{\widetilde{A}}\left(\alpha_{0}\right)+\epsilon_{0}$. With out loss of generality, we may assume that $\left\{\alpha_{n}\right\}_{n \in \mathbb{N}}$ is an increasing sequence. Since $\psi_{\widetilde{A}}$ is a monotonic function, there exists an increasing sequence $\left\{\alpha_{n}^{\prime}\right\}_{n \in \mathbb{N}}$ converging to $\alpha_{0}$ such that $\alpha_{n}^{\prime} \leq \alpha_{n}$, for each $n \in \mathbb{N}$, and $\psi_{\widetilde{A}}$ is continuous on the set $\left\{\alpha_{n}^{\prime} \mid n \in \mathbb{N}\right\}$. Let also $\left\{\beta_{n}\right\}_{n \in \mathbb{N}}$ be a decreasing sequence converging to $\alpha_{0}$ which consist of continuity points of $\psi_{\widetilde{A}}$. Then

$$
\begin{aligned}
\phi_{\widetilde{A}}\left(\alpha_{n}^{\prime}\right) & =\psi_{\widetilde{A}}\left(\alpha_{n}^{\prime}\right) \geq \psi_{\widetilde{A}}\left(\alpha_{n}\right) \geq \psi_{\widetilde{A}}\left(\alpha_{0}\right)+\epsilon_{0}>\psi_{\widetilde{A}}\left(\alpha_{0}\right) \\
& \geq \psi_{\widetilde{A}}\left(\beta_{n}\right)=\phi_{\widetilde{A}}\left(\beta_{n}\right),
\end{aligned}
$$

for each $n \in \mathbb{N}$. Hence

$\forall n \in \mathbb{N}, \quad \phi_{\widetilde{A}}\left(\alpha_{n}^{\prime}\right)-\phi_{\widetilde{A}}\left(\beta_{n}\right)>\epsilon_{0}$,

which implies that $\phi_{\widetilde{A}}$ is also discontinuous at this point.

Proof of Lemma 3 Assume $\widetilde{\tau}_{1} \prec \widetilde{\tau}_{2}$. Note that, from Definition $3, \widetilde{\pi}_{\tilde{\varphi}}\left(\widetilde{\tau}_{1}\right) \preceq \widetilde{\pi}_{\widetilde{\varphi}}\left(\widetilde{\tau}_{2}\right)$ if and only if $\left(\widetilde{\pi}_{\tilde{\varphi}}\left(\widetilde{\tau}_{1}\right)\right)_{\alpha}^{L} \leq\left(\widetilde{\pi}_{\tilde{\varphi}}\left(\widetilde{\tau}_{2}\right)\right)_{\alpha}^{L}$ and $\left(\widetilde{\pi}_{\tilde{\varphi}}\left(\widetilde{\tau}_{1}\right)\right)_{\alpha}^{U} \leq\left(\widetilde{\pi}_{\tilde{\varphi}}\left(\widetilde{\tau}_{2}\right)\right)_{\alpha}^{U}$ for all $\alpha \in[0,1]$. Now, since $\tilde{\tau}_{1} \prec \widetilde{\tau}_{2}$, we have $\left(\tilde{\tau}_{1}\right)_{\alpha}<\left(\tilde{\tau}_{1}\right)_{\alpha}$ for all $\alpha \in[0,1]$. On the other hand, due to the assumption and MLR property of the underlying family of density function, we know that $E_{\left(\tau^{*}\right)_{\beta}}\left[\varphi\left(\widetilde{\mathbf{X}}_{\beta}\right)\right]$ is a non-decreasing function of $\widetilde{\tau}_{\beta}$ for every $\beta \in[0,1]$. So for every $\alpha \in[0,1]$, we have

$$
\begin{aligned}
\left(\tilde{\pi}_{\tilde{\varphi}}\left(\widetilde{\tau}_{1}\right)\right)_{\alpha}^{L} & =\inf _{\beta \geq \alpha} E_{H^{\beta}: \tau_{\beta}=\left(\widetilde{\tau}_{1}\right)_{\beta}}\left(\varphi\left(\widetilde{\mathbf{X}}_{\beta}\right)\right) \\
& \leq \inf _{\beta \geq \alpha} E_{H^{\beta}: \widetilde{\tau}_{\beta}=\left(\widetilde{\tau}_{2}\right)_{\beta}}\left(\varphi\left(\widetilde{\mathbf{X}}_{\beta}\right)\right) \\
& =\left(\widetilde{\pi}_{\widetilde{\varphi}}\left(\widetilde{\tau}_{2}\right)\right)_{\alpha}^{L},
\end{aligned}
$$

and

$$
\begin{aligned}
\left(\widetilde{\pi}_{\tilde{\varphi}}\left(\widetilde{\tau}_{1}\right)\right)_{\alpha}^{U} & =\sup _{\beta \geq \alpha} E_{H^{\beta}: \widetilde{\tau}_{\beta}=\left(\widetilde{\tau}_{1}\right)_{\beta}}\left(\varphi\left(\widetilde{\mathbf{X}}_{\beta}\right)\right) \\
& \leq \sup _{\beta \geq \alpha} E_{H^{\beta}: \widetilde{\tau}_{\beta}=\left(\widetilde{\tau}_{2}\right)_{\beta}}\left(\varphi\left(\widetilde{\mathbf{X}}_{\beta}\right)\right) \\
& =\left(\widetilde{\pi}_{\widetilde{\varphi}}\left(\widetilde{\tau}_{2}\right)\right)_{\alpha}^{U},
\end{aligned}
$$

which is the desired result.

\section{References}

Akbari MG, Rezaei A (2009) Statistical inference about the variance of fuzzy random variables. Sankhyā Indian J Stat 71.B(Part 2):206221

Akbari MG, Rezaei A (2010) Bootstrap testing fuzzy hypotheses and observations on fuzzy statistic. Expert Syst Appl 37:5782-5787

Arefi M, Taheri SM (2011) Testing fuzzy hypotheses using fuzzy data based on fuzzy test statistic. J Uncertain Syst 5:45-61

Arnold BF (1996) An approach to fuzzy hypothesis testing. Metrika 44(1):119-126

Arnold BF (1998) Testing fuzzy hypothesis with crisp data. Fuzzy Sets Syst 94(3):323-333

Bertoluzza C, Gil MA, Ralescu DA (2002) Statistical modeling, analysis and management of fuzzy data. Physica-Verlag, Heidelberg

Buckley JJ (2006) Fuzzy statistics; studies in fuzziness and soft computing. Springer, Berlin

Chachi J, Taheri SM (2011) Fuzzy confidence intervals for mean of Gaussian fuzzy random variables. Expert Syst Appl 38:5240-5244

Denœux T, Masson MH, Herbert PH (2005) Non-parametric rank-based statistics and significance tests for fuzzy data. Fuzzy Sets Syst 153:1-28

Dubois D, Prade H (1983) Ranking of fuzzy numbers in the setting of possibility theory. Inf Sci 30:183-224 
Elsherif AK, Abbady FM, Abdelhamid GM (2009) An application of fuzzy hypotheses testing in radar detection. In: 13th international conference on aerospace science and aviation technology, ASAT13 , pp 1-9

Filzmoser P, Viertl R (2004) Testing hypotheses with fuzzy data: the fuzzy p-value. Metrika 59:21-29

Gil MA, Montenegro M, Rodríguez G, Colubi A, Casals MR (2006) Bootstrap approach to the multi-sample test of means with imprecise data'. Comput Stat Data Anal 51:148-162

Gonzalez-Rodríguez G, Montenegro M, Colubi A, GilMA (2006) Bootstrap techniques and fuzzy random variables: Synergy in hypothesis testing with fuzzy data. Fuzzy Sets Syst 157:2608-2613

Grzegorzewski P (1998) Statistical inference about the median from vague data. Control Cybern 27:447-464

Grzegorzewski P (2000) Testing statistical hypotheses with vague data. Fuzzy Sets Syst 11:501-510

Grzegorzewski P (2004) Distribution-free tests for vague data. In: Lopez-Diaz M et al (eds) Soft methodology and random information systems. Springer, Heidelberg, pp 495-502

Grzegorzewski P (2005) Two-sample median test for vague data. In: Proceedings of the 4th conference European society for fuzzy logic and technology-Eusflat, Barcelona, pp 621-626

Grzegorzewski P (2008) A bi-robust test for vague data. In: Magdalena L, Ojeda-Aciego M, Verdegay JL (eds) Proceedings of the 12th international conference on information processing and management of uncertainty in knowledge-based systems, IPMU2008, Spain, Torremolinos (Malaga), June 22-27, pp 138-144

Grzegorzewski P (2009) K-sample median test for vague data. Int J Intell Syst 24:529-539

Geyer C, Meeden G (2005) Fuzzy and randomized confidence intervals and p-values. Stat Sci 20:358-366

Hesamian G, Taheri SM (2013) Fuzzy empirical distribution: properties and applications. Kybernetika 49:962-982

Hesamian G, Chachi J (2013) Two-sample KolmogorovSmirnov fuzzy test for fuzzy random variables. Stat Pap. doi:10.1007/ s00362-013-0566-2

Hryniewicz O (2006a) Goodman-Kruskal measure of dependence for fuzzy ordered categorical data. Comput Stat Data Anal 51:323334

Hryniewicz O (2006b) Possibilistic decisions and fuzzy statistical tests. Fuzzy Sets Syst 157:2665-2673

Kahraman C, Bozdag CF, Ruan D (2004) Fuzzy sets approaches to statistical parametric and non-parametric tests. Int J Intell Syst 19:1069-1078
Kruse R, Meyer KD (1987) Statistics with vague data. D. Riedel Publishing, New York

Kwakernaak H (1978) Fuzzy random variables, part I: definitions and theorems. Inf Sci 19:1-15

Kwakernaak H (1979) Fuzzy random variables, part II: algorithms and examples for the discrete case. Inf Sci 17:253-278

Lee KH (2005) First course on fuzzy theory and applications. Springer, Berlin

Lehmann EL, Romano JP (2005) Testing statistical hypotheses, 3rd edn. Springer, New York

Liu B (2004) Uncertainty theory. Springer, Berlin

Lin P, Wu B, Watada J (2010) Kolmogorov-Smirnov two sample test with continuous fuzzy data. Adv Intell Soft Comput 68:175-186

Montenegro M, Casals MR, Lubiano MA, Gil MA (2001) Two-sample hypothesis tests of means of a fuzzy random variable. Inf Sci 133:89-100

Montenegro M, Colubi A, Casals MR, Gil MA (2004) Asymptotic and Bootstrap techniques for testing the expected value of a fuzzy random variable. Metrika 59:31-49

Nguyen H, Wu B (2006) Fundamentals of statistics with fuzzy data. Springer, Netherlands

Parchami A, Taheri SM, Mashinchi M (2010) Fuzzy p-value in testing fuzzy hypotheses with crisp data. Stat Pap 51:209-226

Shao J (2003) Mathematical statistics. Springer, New York

Taheri SM, Arefi M (2009) Testing fuzzy hypotheses based on fuzzy test statistic. Soft Comput 13:617-625

Taheri SM, Behboodian J (1999) Neyman-Pearson lemma for fuzzy hypothesis testing. Metrika 49:3-17

Taheri SM, Behboodian J (2001) A Bayesian approach to fuzzy hypotheses testing. Fuzzy Sets Syst 123:39-48

Taheri SM, Hesamian G (2011) Goodman-Kruskal measure of association for fuzzy-categorized variables. Kybernetika 47:110-122

Taheri SM, Hesamian G (2012) A generalization of the Wilcoxon signed-rank test and its applications. Stat Pap 54:457-470

Torabi H, Behboodian J, Taheri SM (2006) Neyman-Pearson lemma for fuzzy hypothesis testing with vague data. Metrika 64:289-304

Viertl R (2006) Univariate statistical analysis with fuzzy data. Comput Stat Data Anal 51:133-147

Viertl R (2011) Statistical methods for fuzzy data. Wiley, Chichester

Wu HC (2005) Statistical hypotheses testing for fuzzy data. Inf Sci 175:30-57

Zadeh LA (1965) Fuzzy sets. Inf Control 8:338-353 